\title{
PIPERACILLIN/TAZOBACTAM IN CONTINUOUS INFUSION VERSUS INTERMITTENT INFUSION IN CHILDREN WITH FEBRILE NEUTROPENIA
}

\author{
Fortino Solórzano-Santos ${ }^{1}$, Anai Quezada-Herrera ${ }^{2}$, Yazmín Fuentes-Pacheco ${ }^{3}$, María G. Labra- \\ Zamora $^{3}$, Genoveva Rodríguez- Coello ${ }^{4}$, Carlos E. Aguirre-Morales ${ }^{5}$, Dassaev Izelo-Flores ${ }^{6}$, Onofre \\ Muñoz- Hernández ${ }^{7}$, and María G. Miranda-Novales ${ }^{8}$ \\ ${ }^{1}$ Research Unit in Evidence-Based Medicine, Hospital Infantil De Mexico "Federico Gómez", Mexico City; ${ }^{2}$ Hospital \\ del Niño y del Adolescente Morelense, Morelos; ${ }^{3}$ High Specialty Medical Unit, Hospital de Pediatría, CMN SXXI, \\ Instituto Mexicano del Seguro Social, Mexico City; ${ }^{4}$ Hospital para el Niño Poblano, Secretaría de Salud, Puebla de \\ Zaragoza, Puebla; ${ }^{5}$ Centro Médico ABC, Mexico City; ${ }^{6}$ Hospital General de Zona No. 20, Instituto Mexicano del Seguro \\ Social, Puebla; ${ }^{7}$ Research Directorate, Hospital Infantil de México "Federico Gómez", SSA, Mexico City; ${ }^{8}$ Analysis \\ and Synthesis of Evidence Research Unit, Coordinación de Investigación en Salud, Instituto Mexicano del Seguro Social, \\ Mexico City, Mexico.
}

\begin{abstract}
Background: Febrile neutropenia (FN) is a common complication in children who receive chemotherapy for cancer. Objective: The objective of this study was to evaluate the clinical efficacy of the continuous versus intermittent infusion of piperacillin/ tazobactam (TZP) in febrile neutropenic pediatric patients. Methods: This is a non-blinded randomized controlled clinical trial. Eligible group consisted of hemato-oncological patients with FN who were candidates to receive TZP. Patients were randomized to one of two groups: Group 1 received antibiotic treatment through intravenous intermittent infusion of TZP $300 \mathrm{mg} / \mathrm{kg} / \mathrm{day}$ based on piperacillin, divided into four doses, not exceeding 16 g/day; Group 2 received an initial TZP loading dose of 75 mg/kg infusion over $30 \mathrm{~min}$, and then a continuous infusion of TZP $300 \mathrm{mg} / \mathrm{kg} /$ day through central line with pump over 24 h. Results: There were 176 episodes that could be assessed, 100 in Group 1 and 76 in Group 2. There was no statistically significant difference in treatment failure in the experimental group (continuous infusion) compared with the intermittent group, $21 \%$ versus $13 \%(p=0.15)$. The increase in the absolute risk reduction was $0.08 \%$ ( $95 \%$ confidence interval $0.12-0.30$ ), and the number needed to treat was 12.4. One patient in each group died. Conclusions: There were no differences in fever resolution, clinical cure rate, or mortality when comparing the continuous with the intermittent TZP infusion. (REV INVEST CLIN. 2019;71:283-90)
\end{abstract}

Key words: Beta-lactams. Febrile neutropenia. Infusion. Piperacillin-tazobactam.

\author{
Corresponding author: \\ * María Guadalupe Miranda-Novales \\ Unidad de Investigación en Análisis \\ y Síntesis de la Evidencia \\ Coordinación de Investigación en Salud \\ Instituto Mexicano del Seguro Social \\ Av. Cuauhtémoc, 330 \\ Col. Doctores, Del. Cuauhtémoc \\ C.P. 06720, Mexico City, Mexico \\ E-mail: guadalumiranda@outlook.es
}

Received for publication: 10-12-2018

Approved for publication: 23-04-2019

DOI: $10.24875 / R I C .19002943$ 


\section{INTRODUCTION}

Febrile neutropenia (FN) is a common complication in children who receive chemotherapy for cancer. Fever may be the only indicator of a severe infection and the prompt needs for antimicrobial treatment ${ }^{1}$. According to the current guidelines, initial empirical antimicrobial therapy should cover enteric Gram-negative microorganisms and Pseudomonas aeruginosa ${ }^{2,3}$; in special conditions, coverage for Gram-positive cocci should be included. Monotherapy with an antipseudomonas $\beta$-lactam, a carbapenem, or a fourthgeneration cephalosporin has been recommended as the first-line therapy ${ }^{3}$. In pediatric oncology patients, infections by antibiotic-resistant $P$. aeruginosa are not common, although the clinical course can be devastating, and thus, some authors suggest antimicrobial combinations ${ }^{4}$. Increased prevalence of antimicrobial-resistant infections with Gram-negative bacteria has been reported in children, associated to a higher mortality rate ${ }^{2}$. Empirical antimicrobial treatment in these patients is based on different conditions such as clinical patient characteristics, local resistance patterns, and drug availability. It is of utmost importance that antibiotics achieve optimal tissue concentration in the pediatric population. It has been described that physiological changes in children with cancer in critical conditions can affect the pharmacokinetics of antibiotics, increasing the risk of failure to achieve adequate antimicrobial concentrations, and thus suppress the growth of resistant bacteria. Suboptimal antimicrobial dosing may be associated with poorer clinical outcomes ${ }^{5}$.

Piperacillin/tazobactam (TZP) is a beta-lactam/ beta-lactamase inhibitor combination recommended as empirical treatment for pediatric FN patients. The $\beta$-lactam antibiotics are characterized by having a short elimination half-life, which conditions their administration schedule by brief intermittent infusions several times a day. For these antibiotics, a relationship exists between the killing of bacteria and the time that free drug concentration remains above the minimum inhibitory concentration (MIC) against a pathogen. Extending the duration of the infusion is one way to increase the free drug concentrations above the MIC, without using more drug per day. Extended or continuous infusion administration of TZP has been proposed to achieve the best inhibitory concentrations and obtain optimal pharmacodynamics exposures ${ }^{6-8}$. Due to the low number of circulating neutrophils in children with $\mathrm{FN}$, they depend to a large extent on optimal bactericidal levels of antibiotics.

In our hospital, hemato-oncological patients with FN receive piperacillin/tazobactam monotherapy as empirical treatment since 2007. This treatment was selected due to the high frequency of extended-spectrum $\beta$-lactamase-producing (ESBL) enterobacteria, as a strategy to decrease the selective resistance pressure by ceftazidime and limit their persistence and spread. Considering the pharmacokinetic aspects described in children with cancer ${ }^{6}$, the objective of this study was to evaluate the clinical efficacy of the continuous infusion versus the intermittent administration of TZP in FN pediatric patients.

\section{METHODS}

A non-blinded randomized controlled clinical trial was conducted in a third-level pediatric hospital. The eligible group was hemato-oncological pediatric patients ( $\leq 17$ years old), were presented at the hospital with an event of $\mathrm{FN}$, and was candidates to receive empirical antimicrobial treatment with TZP. FN was defined as a single oral temperature of $\geq 38.3^{\circ} \mathrm{C}$, or a temperature of $\geq 38.0^{\circ} \mathrm{C}$ sustained over $1 \mathrm{~h}$ and an absolute neutrophil count (ANC) $<500$ cells $/ \mathrm{mm}^{3}$, or an ANC that is expected to decrease to $<500$ cells $/ \mathrm{mm}^{3}$ over the next $48 \mathrm{~h}$. The study period included from April 2012 to August 2015. Exclusion criteria: Chemotherapy-associated nephrotoxicity, suspected intravascular device-associated infections, a recent event ( $<1$ month) of methicillin-resistant Staphylococcus aureus or Streptococcus pneumoniae invasive infection, history of allergic reaction to any $\beta$-lactam, a FN episode 30 days before present admission, patients with severe sepsis or septic shock, and those in palliative care were excluded from the study. Elimination criteria: Chemotherapy-associated nephrotoxicity during the hospital stay while receiving antimicrobial treatment, violations of the protocol, patients with fungal or viral infections as a cause of fever, those with isolation of Gram-positive cocci or multiresistant Gram-negative bacilli, patients with localized infections (pneumonia, neutropenic colitis, skin and soft tissue infection, and anal fissures/perianal abscesses) that lead to changes in the antimicrobial 
treatment in the first $72 \mathrm{~h}$, and patients in the continuous infusion group in whom antibiotic infusion was suspended for more than $4 \mathrm{~h}$.

The study was approved by the institutional ethical committee (number R-2012-3603-14), and informed consent was obtained from the study participants (parents or legal guardians). After parents signed the informed consent form and children the assent form, patients were allocated according to a computergenerated randomization list. Group 1 received antibiotic treatment through an intravenous intermittent infusion of TZP $300 \mathrm{mg} / \mathrm{kg} /$ day based on piperacillin, divided into four doses, not exceeding $16 \mathrm{~g} /$ day (diluted $50 \mathrm{mg} / \mathrm{mL}$ in $5 \%$ glucose solution over $30 \mathrm{~min}$ ) and Group 2 received an initial TZP loading dose of $75 \mathrm{mg} / \mathrm{kg}$ infusion over $30 \mathrm{~min}$ (diluted $50 \mathrm{mg} / \mathrm{mL}$ in $5 \%$ glucose solution), and then a continuous infusion of TZP $300 \mathrm{mg} / \mathrm{kg} /$ day (diluted $25 \mathrm{mg} / \mathrm{mL}$ in $5 \%$ glucose solution) through central line with pump over $24 \mathrm{~h}$. Before initiating the protocol, several sessions were conducted with the nursing staff to inform the study procedures. Preparation, administration schedule, and correct infusion rate in both treatment modalities were supervised.

Clinical evaluation was made at admission and every $24 \mathrm{~h}$ by two researchers (QHA and FPY). On hospital admission, each patient underwent hematic biometry, C-reactive protein (CRP), blood chemistry, and two peripheral blood cultures (taken from two different venipuncture sites, each 30 min apart) before starting antimicrobial treatment. Blood cultures were processed by BACTEC system (Becton-Dickinson). Growth bacteria and yeast were identified by the VITEK 2 system (bioMérieux).

FN episodes were classified as microbiologically documented infections (MDI), clinically documented infection, and fever of unknown origin (FUO). Outcomes: Clinical cure, when fever subsided within $96 \mathrm{~h}$ after starting antimicrobial treatment, or if clinical signs of infection resolved and the patient was discharged after completing the antimicrobial scheme. Treatment failure, if fever persisted and a modification of the initial empirical treatment was done, or if the patient died because of the infection. A protocol violation was defined as a modification of the empirical treatment without criteria for treatment failure.
Antibiotic regimens were adjusted according to microbiological culture results, when available, in consultation with an infectious diseases specialist.

\section{Statistical analysis}

Descriptive statistics with simple frequencies and percentages. Comparisons between groups were done with Mantel-Haenszel Chi-square, Fisher's exact test, and Mann-Whitney U-test. Intention-to-treat analysis and per protocol were performed, as well as calculation of absolute risk reduction and number needed to treat. The sample size was calculated to detect a difference of $15 \%$ between the groups, alpha 0.5 , beta 0.20 , and power of $80 \%$; at least 76 patients per group were needed.

\section{RESULTS}

During the study period (April 2012-August 2015), 327 events of FN were evaluated; 86 cases were excluded and 39 refused to participate. A total of 202 episodes were randomized: 112 were assigned to intermittent infusion (Group 1) and 90 to continuous infusion (Group 2). Twelve cases from Group 1 and 14 from Group 2 were eliminated. The causes for elimination were as follows: in Group 1, isolation of a Gram-positive microorganism ${ }^{4}$ and clinical infection that required extended antimicrobial coverage (four perianal abscesses and four pneumonias); in Group 2, the isolation of a resistant microorganism in five (two Gram-positive and three ESBL-producing enterobacteria), clinical infection that required extended antimicrobial coverage (two pneumonias and one neutropenic colitis), and five children without an adequate venous access and suspension of antimicrobial infusion for $>4 \mathrm{~h}$, one with a documented viral infection (herpetic stomatitis), and one due to cisplatin-induced tubulopathy (Fig. 1).

There were 176 episodes of FN that could be evaluated, 100 in Group 1 and 76 in Group 2. The male gender was more frequent, $57.3 \%$ and $61.8 \%$ in each group. Median age was 9 and 10 years old (1-16 year) for Group 1 and Group 2, respectively. There were $34(19.3 \%)$ episodes in patients with acute leukemia (24 acute lymphoblastic leukemia and 10 acute myeloblastic leukemia) and $142(80.6 \%)$ with different neoplasias (solid tumors in 76 , non-Hodgkin 
Figure 1 . Study design flow diagram.

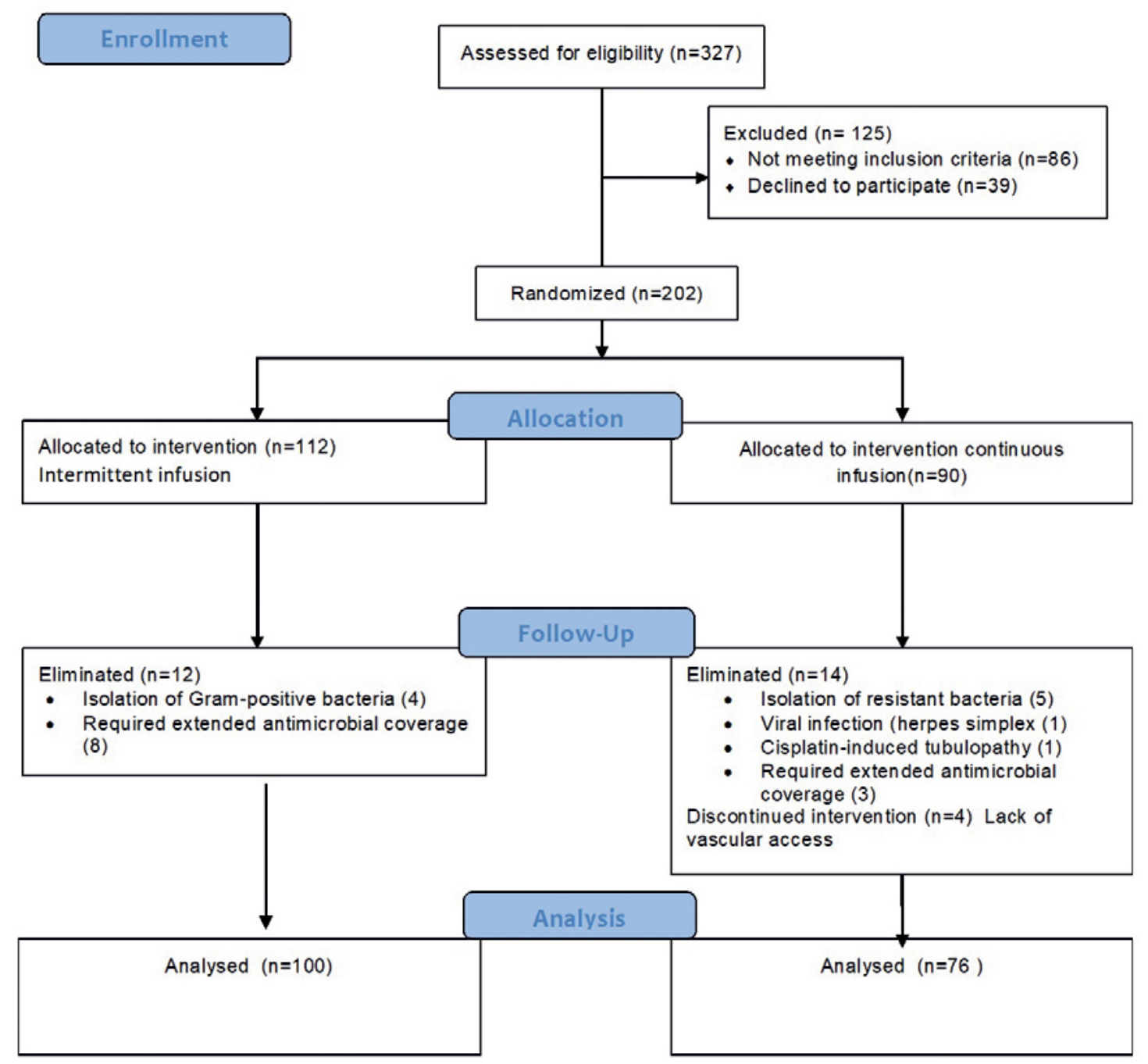

lymphoma in 40, central nervous system tumors in 22, and malignant histiocytosis in 4). Disease distributions in groups were as follows: in Group 1, solid tumors were $83 \%$ and acute leukemia and lymphomas (nonHodgkin and Burkitt type), 17\%; in Group 2, solid tumors were $75 \%$ and leukemia and lymphomas (nonHodgkin and Burkitt type) $25 \%(p=0.20)$. There were no statistically significant differences in gender, age, type of cancer, comorbidity, neutrophil count and CRP values on admission, filgrastim administration, and intensity of fever (only $15 \%$ had $>39.1^{\circ} \mathrm{C}$ ).

There were no statistically significant differences in clinical characteristics of FN episodes between groups (Table 1 ). There were $9 \%$ and $6.5 \%$ of $\mathrm{MDI}$ (bacteremia) in Group 1 and Group 2, respectively. Most of the episodes corresponded to FUO (81\%). Fever decreased in the first $48 \mathrm{~h}$ after starting antibiotic therapy in $45 \%$ of patients. Improvement of signs and symptoms of infection at $72 \mathrm{~h}$ was similar in both groups ( $80 \%$ and $73 \%$ ). There were 13 (13\%) failures in Group 1 (six corresponded to MDI, five were clinically documented, and two were due to FUO), and 16 (21\%) in Group 2 (four corresponded to $\mathrm{MDI}$, eight to clinically documented, and four due to FUO). The difference was not statistically significant. One patient in each group died: in Group 1, one patient from septic shock due to Bacillus spp., and in Group 2, one patient with neutropenic enterocolitis (Table 2). 
Table 1. General characteristics of febrile neutropenia episodes

\begin{tabular}{|c|c|c|c|}
\hline Variable & $\begin{array}{c}\text { Group } 1 \text { intermittent } \\
\text { TZP infusion } \\
(n=100)\end{array}$ & $\begin{array}{l}\text { Group } 2 \text { continuous } \\
\text { TZP infusion } \\
(n=76)\end{array}$ & p value* \\
\hline \multicolumn{4}{|l|}{ Gender } \\
\hline Male & 56 & 47 & 0.61 \\
\hline Female & 44 & 29 & \\
\hline Age (median, interval) & $9(1-16)$ & $10(1-16)$ & $0.69^{* *}$ \\
\hline \multicolumn{4}{|l|}{ Type of cancer } \\
\hline Solid tumor & 83 & 57 & 0.20 \\
\hline Leukemia/lymphoma & 17 & 19 & \\
\hline \multicolumn{4}{|l|}{ Comorbidity } \\
\hline Yes & 5 & 10 & 0.97 \\
\hline No & 95 & 66 & \\
\hline \multicolumn{4}{|l|}{ Degree of neutropenia } \\
\hline $0-100 \mathrm{cell} / \mathrm{mm}^{3}$ & 69 & 58 & 0.28 \\
\hline $101-500 \mathrm{cell} / \mathrm{mm}^{3}$ & 31 & 18 & \\
\hline \multicolumn{4}{|l|}{ C-reactive protein } \\
\hline$<90 \mathrm{mg} / \mathrm{L}$ & 57 & 40 & 0.67 \\
\hline$>91 \mathrm{mg} / \mathrm{L}$ & 43 & 36 & \\
\hline \multicolumn{4}{|l|}{ Filgrastim administration } \\
\hline Yes & 65 & 45 & 0.43 \\
\hline No & 35 & 31 & \\
\hline \multicolumn{4}{|l|}{ Fever at admission } \\
\hline $38-39^{\circ} \mathrm{C}$ & 88 & 61 & 0.15 \\
\hline$>39.1^{\circ} \mathrm{C}$ & 12 & 15 & \\
\hline
\end{tabular}

*Chi-square; ${ }^{* *}$ Mann-Whitney U-test

Enterobacteriaceae and other Gram-negative bacteria were the most frequent isolates (Table 3). All $P$. aeruginosa isolates were TZP susceptible, but in one patient (in the continuous infusion group), TZP was changed to a carbapenem due to fever persistence at day 4. In patients with ESBL-producing Escherichia coli and Enterobacter cloacae isolates, antimicrobial treatment was also changed to a carbapenem. In those patients with Salmonella enteritidis isolates, treatment was modified for cefotaxime.

With the failures in the experimental group (continuous infusion) and the intermittent group (21\% vs. $13 \%$ ), the increase in the absolute risk reduction was $0.08 \%$ (95\% confidence interval [Cl]: 0.03-0.19), and the number needed to treat was 12.4. In the intention-to-treat analysis, assuming that all patients lost or eliminated from the control and the experimental group had the same (acceptable) outcome, the absolute risk reduction was $0.06(95 \% \mathrm{Cl} 0.04$ 0.177 ) and the number needed to treat was 16.4 patients.

\section{DISCUSSION}

Fever can be the expression of the beginning of a potentially severe infection in cancer patients receiving chemotherapy. An early diagnosis and initiation of the appropriate antimicrobial treatment could be 
Table 2. Type of infection and treatment outcomes of febrile neutropenia episodes

\begin{tabular}{|c|c|c|c|}
\hline Infections and outcome & $\begin{array}{l}\text { Group } 1 \text { intermittent } \\
\text { TZP infusion } \\
(n=100)\end{array}$ & $\begin{array}{c}\text { Group } 2 \text { continuous } \\
\text { TZP infusion } \\
(n=76)\end{array}$ & $p$ value \\
\hline \multicolumn{4}{|l|}{ Clinical diagnosis of infection } \\
\hline Microbiologically documented & 9 & 5 & 0.84 \\
\hline Clinically documented & 7 & 11 & \\
\hline Fever of unknown origin & 84 & 60 & \\
\hline \multicolumn{4}{|l|}{ Improvement at $72 \mathrm{~h}$} \\
\hline Yes & 80 & 56 & 0.32 \\
\hline No & 20 & 20 & \\
\hline Treatment failure & 13 & 16 & 0.15 \\
\hline Deaths & 1 & 1 & 0.67 \\
\hline
\end{tabular}

lifesaving. According to the current clinical guidelines, these patients need to receive empirical antimicrobial treatment, including an antipseudomonal $\beta$-lactam or a carbapenem ${ }^{3,4,6}$. TZP has been recommended as a suitable antibiotic in children ${ }^{9}$. With standard doses of $\beta$-lactams administered in intermittent infusion, a high peak concentration is attained, but a short halflife leads to a fall in plasma drug levels and suboptimal free drug concentration above the MIC (Ft > MIC) against certain pathogens. On the other hand, a prolonged (extended or continuous) antibiotic infusion offers more consistent plasma drug levels and maximizing of $\mathrm{fT}>\mathrm{MIC}^{6}$. Some studies using extended infusion TZP dosing strategies in adult population suggest best reaching of pharmacodynamic targets than with traditional intermittent dosing, and it has been associated with improved clinical outcomes ${ }^{7,10-12}$. There are fewer reports that explored this strategy in pediatric population; however, implementing extended infusion TZP as standard of care to pediatric patients is suggested to be achievable $6,13,14$.

In this study, we explored the efficacy of continuous TZP infusion in febrile neutropenic children, and we found that there were no differences in fever resolution, clinical cure rate, and mortality when comparing with intermittent infusion. Results of a recent study comparing extended infusion ( $4 \mathrm{~h}$ ) of $\beta$-lactams versus bolus administration in FN found that extended infusion was superior (74\% vs. $55 \%$ ) in terms of overall response ${ }^{1} 5$. Most of the studies have not demonstrated statistically significant differences in clinical outcomes and mortality ${ }^{16,17}$, but several authors highlighted the reduction in costs ${ }^{18}$.

Limitations of this study include a low frequency of episodes with proven bacteremia (8\%); some other studies in $\mathrm{FN}$ patients showed that this condition does not exceed $15 \%{ }^{19}$. Results in this study are within the range of several reports. In this study, in about $45 \%$ of the cases, the fever decreased in the first $48 \mathrm{~h}$ of the start of the antibiotic treatment; some authors proposed a limit of $72 \mathrm{~h}$ to consider efficacy attributable to the antibiotic, although the average duration of fever is 3-4 days ${ }^{20}$. It is very likely that patients with fever lasting 24-36 h do not need antimicrobial treatment. Santolaya et al. have proposed to withhold antimicrobial treatment in children with cancer, fever, and neutropenia if a respiratory infection is present until a result of a polymerase chain reaction microarray for 17 respiratory viruses confirm a viral infection ${ }^{21}$; in our study, most of the patients had FUO. Some authors have ruled out the importance of the administration time for the first dose of antimicrobial in children with $\mathrm{FN}^{22}$. Until rapid and accurate microbiological diagnostic methods are available, cancer patients will continue to receive antimicrobial schemes for their safety. Despite having a higher frequency of $P$. aeruginosa bacteremia episodes in our study (one-third of the isolates), the outcome was not associated with death, contrary to $38.9 \%$ lethality in febrile neutropenic children and adolescents with $P$. aeruginosa bacteremia reported by Kim et al. $^{4}$; furthermore, $P$. aeruginosa strains did not have a multidrug resistance profile. 
Table 3. Microorganisms isolated from blood in febrile neutropenia episodes

\begin{tabular}{lcc}
\hline Microorganism & $\begin{array}{c}\text { Group } 1 \\
\text { intermittent infusion } \\
(\mathrm{n}=9)\end{array}$ & $\begin{array}{c}\text { Group } 2 \\
\text { continuous infusion } \\
(\mathrm{n}=5)\end{array}$ \\
\hline Pseudomonas aeruginosa & 4 & 1 \\
Klebsiella oxytoca & 1 & 0 \\
Salmonella enteritidis & 2 & 0 \\
Escherichia coli & 1 & 1 \\
Escherichia coli ESBL+* & 0 & 1 \\
Enterobacter cloacae & 1 & 1 \\
Klebsiella pneumoniae ESBL+ & 0 & 1 \\
\hline
\end{tabular}

*ESBL+: Extended-spectrum $\beta$-lactamase producer.

Due to difficulties to ensure an adequate intravenous access in this study, four patients in the continuous infusions group were eliminated due to suspension of the infusion for $\geq 4 \mathrm{~h}$ and were not included in the analysis, which may constitute a bias. This is one of the limitations of the continuous infusion strategy because a central venous catheter is needed to comply with it; other issues are that the mobility of the patient may be limited and that the administration of non-compatible IV medications could require placing an additional peripheral short catheter ${ }^{14,15}$. To ensure that this strategy is successful, a trained staff and the availability of a pump infusion system are required ${ }^{20}$. These issues could be resolved with an extended infusion administration (3-4 h).

In several studies, this modality proved to be safe and cost effective ${ }^{16-18}$. In this clinical trial, there was no difference in terms of improvement at $72 \mathrm{~h}$ or clinical cure. Results of the absolute risk reduction both in the analysis per protocol (patients who completed the treatment originally allocated), as well as in the intention-to-treat analysis, showed a small benefit in favor of the continuous infusion method. Prolonged infusion dosing strategies allow maintenance of antibiotic concentrations above the MIC against certain pathogens; this treatment modality is being used more frequently in critically ill and high-risk patients with FN. Until now, it is unclear whether prolonged infusions of $\beta$-lactams could translate into better clinical cure and/or survival. The accumulated information points to this being a good strategy. In addition, some studies found that prolonged infusion of TZP reduces the costs of treatment related to the form of administration and decreases dosing errors ${ }^{10,13-15,23,24}$.
In the present study there were no differences in fever resolution, clinical cure rate and mortality comparing the continuous with the intermittent infusion. Prolonged infusions of beta-lactam antibiotics warrant further evaluation in pediatric patients.

\section{REFERENCES}

1. Cohen C, King A, Lin CP, Friedman GK, Monroe K, Kutny M, et al. Protocol for reducing time to antibiotics in pediatric patients presenting to an emergency department with fever and neutropenia: efficacy and barriers. Pediatr Emerg Care. 2016; 32:739-45

2. Lee JH, Kim SK, Kim SK, Han SB, Lee JW, Lee DG, et al. Increase in antibiotic-resistant gram-negative bacterial infections in febrile neutropenic children. Infect Chemother. 2016;48:181-9.

3. Lehrnbecher T, Robinson P, Fisher B, Alexander S, Ammann RA, Beauchemin M, et al. Guideline for the management of fever and neutropenia in children with cancer and hematopoietic stem-cell transplantation recipients: 2017 update. J Clin Oncol. 2017; 35:2082-94.

4. Kim HS, Park BK, Kim SK, Han SB, Lee JW, Lee DG, et al. Clinical characteristics and outcomes of Pseudomonas aeruginosa bacteremia in febrile neutropenic children and adolescents with the impact of antibiotic resistance: a retrospective study. BMC Infect Dis. 2017;17:500.

5. Gonzalez D, Conrado DJ, Theuretzbacher U, Derendorf H. The effect of critical illness on drug distribution. Curr Pharm Biotechnol. 2011;12:2030-6.

6. Cies JJ, Jain J, Kuti JL. Population pharmacokinetics of the piperacillin component of piperacillin/tazobactam in pediatric oncology patients with fever and neutropenia. Pediatr Blood Cancer. 2015;62:477-82.

7. Patel GW, Patel N, Lat A, Trombley K, Enbawe S, Manor K, et al. Outcomes of extended infusion piperacillin/tazobactam for documented gram-negative infections. Diagn Microbiol Infect Dis. 2009;64:236-40.

8. Craig WA. Pharmacokinetic/pharmacodynamic parameters: rationale for antibacterial dosing of mice and men. Clin Infect Dis. 1998;26:1-10

9. Tamura K, Akiyama N, Kanda Y, Saito M. Efficacy and safety of tazobactam/piperacillin as an empirical treatment for the patients of adult and child with febrile neutropenia in Japan. J Infect Chemother. 2015;21:654-62.

10. Yang H, Cui X, Ma Z, Liu L. Evaluation outcomes associated with alternative dosing strategies for piperacillin/tazobactam: a systematic review and meta-analysis. J Pharm Pharm Sci. 2016;19: 274-89.

11. Bao H, Lv Y, Wang D, Xue J, Yan Z. Clinical outcomes of extended versus intermittent administration of piperacillin/tazo- 
bactam for the treatment of hospital-acquired pneumonia: a randomized controlled trial. Eur J Clin Microbiol Infect Dis. 2017; 36:459-66.

12. Fan SY, Shum HP, Cheng WY, Chan YH, Leung SM, Yan WW, et al. Clinical outcomes of extended versus intermittent infusion of piperacillin/tazobactam in critically ill patients: a prospective clinical trial. Pharmacotherapy. 2017:37:109-19.

13. Nichols KR, Knoderer CA, Cox EG, Kays MB. System-wide implementation of the use of an extended-infusion piperacillin/tazobactam dosing strategy: feasibility of utilization from a children's hospital perspective. Clin Ther. 2012;34:1459-65.

14. Knoderer CA, Karmire LC, Andricopulos KL, Nichols KR. Extended infusion of piperacillin/tazobactam in children. J Pediatr Pharmacol Ther. 2017;22:212-7.

15. Ram R, Halavy Y, Amit O, Paran Y, Katchman E, Yachini B, et al. Extended vs bolus infusion of broad-spectrum $\beta$-lactams for febrile neutropenia: an unblinded, randomized trial. Clin Infect Dis. 2018;67:1153-60.

16. Yang $\mathrm{H}$, Zhang C, Zhou Q, Wang Y, Chen L. Clinical outcomes with alternative dosing strategies for piperacillin/tazobactam: a systematic review and meta-analysis. PLoS One. 2015;10: e0116769.

17. Falagas ME, Tansarli GS, Ikawa K, Vardakas KZ. Clinical outcomes with extended or continuous versus short-term intravenous infusion of carbapenems and piperacillin/tazobactam: a systematic review and meta-analysis. Clin Infect Dis. 2013; 56:272-82
18. Brunetti L, Poustchi S, Cunningham D, Toscani M, Nguyen J, Lim J, et al. Clinical and economic impact of empirical extended-infusion piperacillin-tazobactam in a community medical center Ann Pharmacother. 2015;49:754-60.

19. Al-Tawfiq JA, Hinedi K, Khairallah H, Saadeh B, Abbasi S, Noureen $M$, et al. Epidemiology and source of infection in patients with febrile neutropenia: a ten-year longitudinal study. J Infect Public Health. 2019;12:364-6.

20. Horita N, Shibata Y, Watanabe H, Namkoong H, Kaneko T. Comparison of antipseudomonal $\beta$-lactams for febrile neutropenia empiric therapy: systematic review and network meta-analysis. Clin Microbiol Infect. 2017;23:723-9.

21. Santolaya ME, Alvarez AM, Acuña M, Avilés CL, Salgado C, Tordecilla J, et al. Efficacy and safety of withholding antimicrobial treatment in children with cancer, fever and neutropenia, with a demonstrated viral respiratory infection: a randomized clinical trial. Clin Microbiol Infect. 2017;23:173-8.

22. De la Maza $V$, Simian $D$, Castro M, Torres JP, Lucero $Y$, Sepúlveda $F$, et al. Administration time for the first dose of antimicrobials in episodes of fever and neutropenia in children with cancer. Pediatr Infect Dis J. 2015;34:1069-73

23. Maddox ML, DeBoer EC, Hammerquist RJ. Administration of extended infusion piperacillin-tazobactam with the use of smart pump technology. Hosp Pharm. 2014;49:444-8.

24. Wrenn RH, Cluck D, Kennedy L, Ohl C, Williamson JC. Extended infusion compared to standard infusion cefepime as empiric treatment of febrile neutropenia. J Oncol Pharm Pract. 2018;24:170-5 